\title{
Spontaneous Haemoperitoneum in Pregnancy with Placenta Percreta in Bicornuate Uterus with Successful Pregnancy Outcome A case study
}

"Jayasree Santhosh, ${ }^{1}$ Latha V. Kharka, ${ }^{1}$ Atheel Kamona, ${ }^{2}$ Sumaiya Saif Al Amri ${ }^{1}$

\begin{abstract}
Intraperitoneal haemorrhage during pregnancy or postpartum without any history of trauma (spontaneous haemoperitoneum in pregnancy [SHiP]) is a rare condition, causing significant morbidity and mortality for the mother and the neonate. We report a 27 -year-old female patient with SHiP at 31 weeks of gestation who was referred to a tertiary care hospital in Muscat, Oman, in 2019, with right iliac fossa pain, pallor and tachycardia. Radiological investigations revealed intraperitoneal bleeding and a right adnexal haematoma. She was managed conservatively with blood transfusion and supportive care. At 36 weeks of gestation, lower segment caesarean section was done and a live baby with good Apgar score was delivered. Pre-operatively, she was found to have a bicornuate uterus, placenta percreta at the junction of the horns and a right adnexal haematoma. This case highlights the significance of thorough evaluation of acute abdominal pain in pregnancy in avoiding unnecessary surgical intervention and iatrogenic prematurity.
\end{abstract}

Keywords: Abdominal Pain; Caesarean Section; Hemoperitoneum; Placenta Accreta; Pregnancy; Case Report; Oman.

$\mathrm{I}$ NTRAPERITONEAL HAEMORRHAgE DURING PREGNANCY or postpartum (up to 42 days) without any history of trauma is termed spontaneous haemoperitoneum in pregnancy $(\mathrm{SHiP}) .{ }^{1}$ This is a rare condition which can cause significant morbidity and mortality for the mother and neonate. ${ }^{2}$ We present a patient with SHiP who was conservatively managed and delivered at 36 weeks by Caesarean section. The uterus was found to be bicornuate with a placenta percreta at the junction of the horns with possible bleeding from the serosal surface.

\section{Case Report}

A 27-year-old female patient at 31 weeks of gestation (gravida 2 para 1) with history of previous lower segment caesarean section (LSCS), was referred from a regional hospital to a tertiary care hospital in Muscat, Oman, in 2019 for acute abdominal pain. At the regional hospital, she had presented with complaints of right iliac fossa pain. Emergency laparoscopy was planned with a provisional diagnosis of acute appendicitis. However, she refused surgery and hence was referred. She had no significant previous medical illness. On examination, she was found to be pale, tachycardic and tachypnoeic but afebrile. Her haemoglobin had dropped to $8 \mathrm{~g} / \mathrm{dL}$ from $10 \mathrm{~g} / \mathrm{dL}$ over a period of eight hours. Her inflammatory markers were found to be normal and cardiotocography
(CTG) was normal. Abdominal ultrasound scan (USS) revealed free fluid suggestive of haemoperitoneum and a large right adnexal haematoma. Foetal status remained normal. Images were discussed with the radiologist and scar dehiscence was ruled out both clinically and radiologically. There was no obvious inflammatory mass or bowel thickening and the appendix and right kidney looked normal. She was given a blood transfusion and supportive care. On abdominal magnetic resonance imaging (MRI), the haematoma was found to extend from the right iliac fossa to the uterine fundus [Figures 1 and 2]. Abdominal computed tomography (CT) angiography was done as suggested by the consultant surgeon to exclude ongoing haemorrhage. The patient also preferred to proceed with imaging rather than with laparotomy to avoid possible preterm delivery. As there was no active bleeding, she was conservatively managed. She remained stable and became asymptomatic. Followup USS showed that the haematoma was resolving and that there was a marked reduction in free fluid. She was discharged from the hospital with a plan for weekly review with serial growth scans and CTG.

At 36 weeks of gestation, she underwent elective LSCS for breech presentation and delivered a baby weighing $3.2 \mathrm{~kg}$ with good Apgar. Her uterus was found to be partially bicornuate with a shallow external indentation or groove. This had not been documented in her previous caesarean section notes. The placenta 


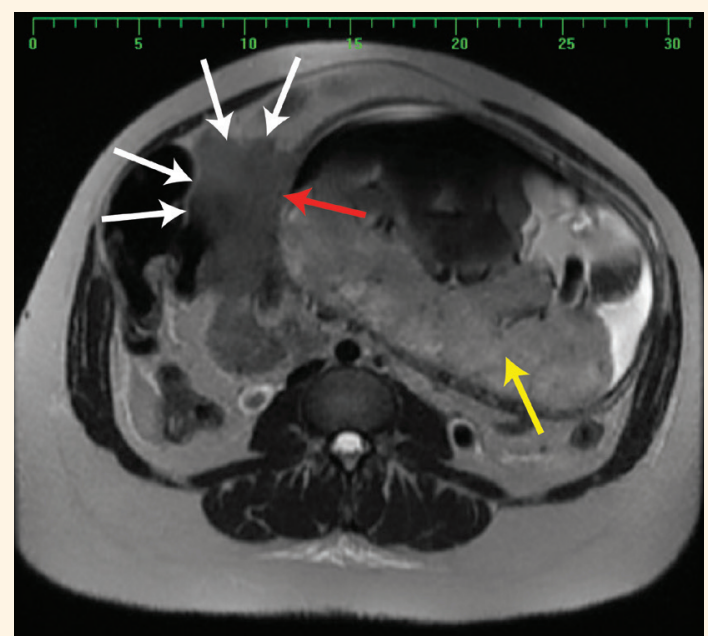

Figure 1: Axial T2 weighted abdominal magnetic resonance image of a 27 -year-old pregnant female patient showing the placenta (yellow arrow) and a welldefined haematoma (white arrows) in the right parauterine region, inseparable from the uterine wall (red arrow).

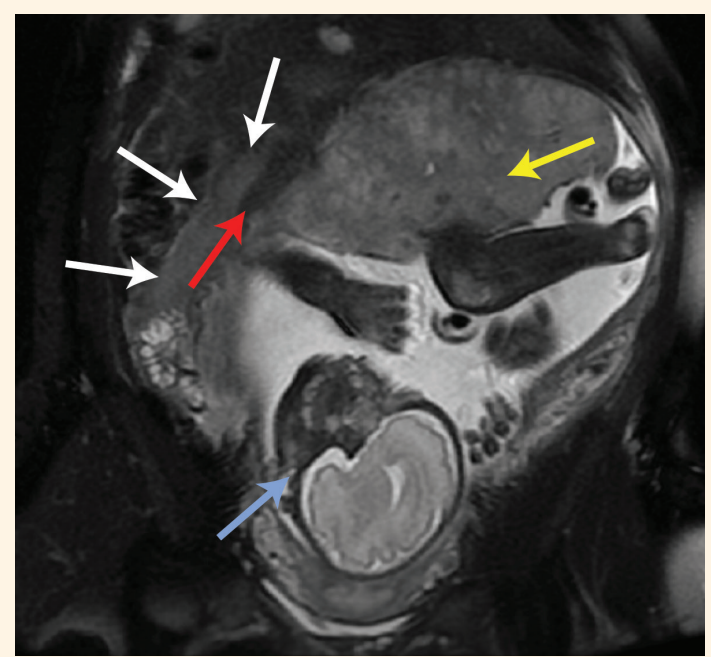

Figure 2: Coronal T2 weighted abdominal magnetic resonance image of a 27 -year-old pregnant female patient showing the placenta (yellow arrow), fetus (blue arrow) and a well-defined haematoma (white arrows) in the right para-uterine region, inseparable from the uterine wall (red arrow).

was found attached to the bridging area between the two horns of the uterus. The delivery of the placenta revealed a myometrial defect covered only by vascular serosa at the junction of the two horns [Figure 3]. As the placenta was infiltrating the serosa, it was assumed to be a case of focal percreta. The previous caesarean scar was found to be intact. The serosa was opened and the uterine defect was closed in layers. The organised necrotic haematoma adherent to the right adnexal region was removed [Figure 4]. Ovaries and appendix looked normal.

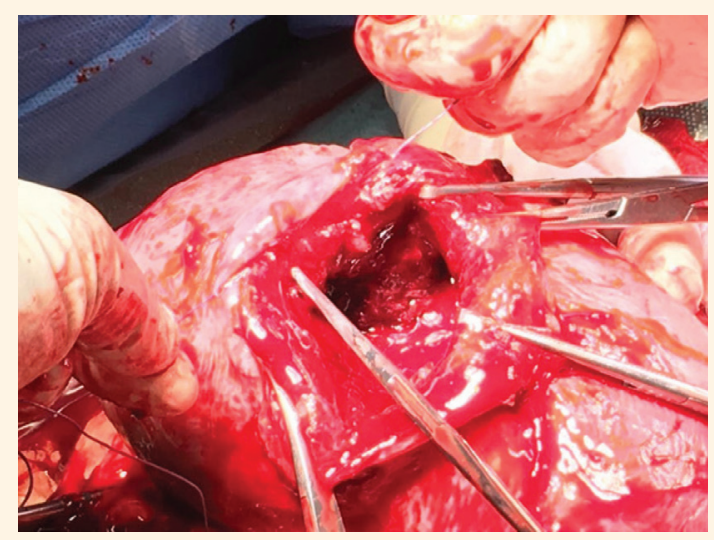

Figure 3: Intraoperative photograph during the lower segment caesarean section of the current patient showing bicornuate uterus with defect in the junction of uterine horns.

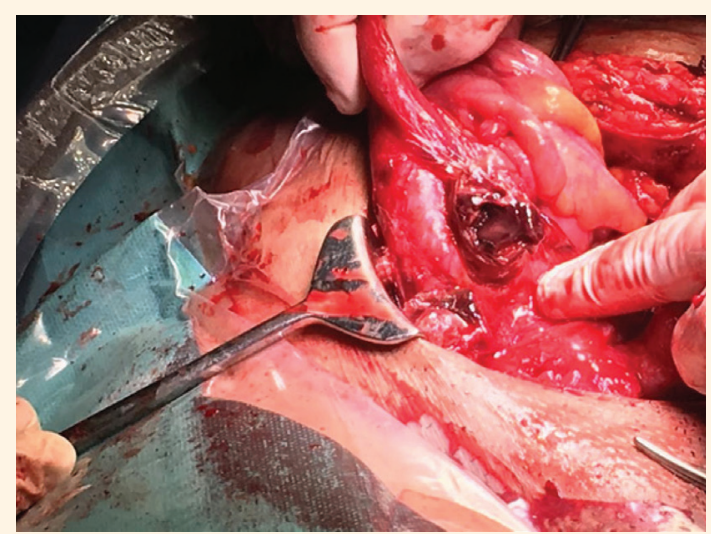

Figure 4: Intraoperative photograph during the lower segment caesarean section of the current patient showing organised right adnexal haematoma.

The patient had an uneventful post-operative recovery and was discharged on day four. Histopathology report showed that the haematoma was surrounded by fibroblastic reaction. A follow-up USS done one year later was found to be normal.

Patient consent was obtained for clinical photography and publication of this case report.

\section{Discussion}

Acute abdominal pain in pregnancy is a diagnostic and therapeutic challenge. Many times the diagnosis is in favour of more common conditions such as appendicitis. The current patient presented with right iliac fossa pain, tachycardia, abdominal tenderness and guarding. However, absence of fever, normal inflammatory markers and a significant drop in haemoglobin was suggestive of intra-abdominal bleeding; this was confirmed by radiological investigations. 
According to a literature review including case reports by $\mathrm{Xu}$ et al., pregnancy-related causes of SHiP are antepartum rupture of uterus, rupture of rudimentary horn, placenta percreta, scar rupture and ruptured corpus luteum of pregnancy. ${ }^{2}$ Causes unrelated to pregnancy are variceal bleed from the surface of uterus, rupture of ovarian vein, venous bleed from the surface of sub-serous myoma, bleeding from endometriotic deposits and rupture of splenic or renal vein. However, the pathogenesis is unclear. Parauterine veins and superficial veins of the uterus, when complicated by endometriosis, can result in bleeding with trivial trauma. This is more so in pregnancy when venous pressure increases due to compression from gravid uterus. Patients present with abdominal pain, nausea, vomiting, pallor, tachycardia, tachypnoea and hypotension. Clinical examination and radiological investigations have limitations during pregnancy.

Lier et al. have done a systematic review and meta-analysis describing 59 cases of SHiP. ${ }^{1}$ In the majority of cases, SHiP occurred in the third trimester of pregnancy and presented with acute abdominal pain, hypovolaemic shock, low haemoglobin and in some cases, foetal distress. Imaging confirmed free peritoneal fluid in $62.7 \%$ cases. At the time of surgery, $91.1 \%$ patients were found to have active bleeding which originated from ruptured uteroovarian vessels (56.8\%), endometriotic implants (21.6\%), haemorrhagic nodules of decidualised cells (2.0\%) or a combination (19.6\%). Median amount of haemoperitoneum was $1600 \mathrm{~mL}$. Of the $76.3 \%$ women who underwent surgical interventions during pregnancy, $15.6 \%$ reported a successful continuation of pregnancy with recurrence rate of $8.5 \%$. There was one maternal death. Perinatal mortality was reported to be $26.9 \%$. They concluded that SHiP is a very serious complication of pregnancy, highly associated with adverse pregnancy outcomes and particularly relevant to women with endometriosis. As there are no preventive measures, early recognition of SHiP plays a crucial role in improving pregnancy outcome.

After reviewing a case series by Lier et al., Van den Bosch published a mini-commentary where he states that in any woman presenting with acute abdominal pain, hypotension and/or foetal distress in the second or third trimester of pregnancy, an abdominal ultrasound examination is to be performed immediately, not only to exclude obstetric causes, but also to look for intra-abdominal free fluid. ${ }^{3}$ In the absence of obstetric causes and other causes of massive abdominal bleeding such as splenic rupture, SHiP should be considered and prompt multidisciplinary intervention is to be started. In their case series, Lier et al. advocates median laparotomy to obtain optimal access to all abdominal compartments. ${ }^{4}$ If the foetus is viable, a caesarean section may be needed to reduce the uterine size for thorough assessment of the posterior uterine wall and posterior compartment. The bleeding point(s) are to be repaired by suturing and/or the use of haemostatic agents. Hysterectomy was not required for any patient in their case series. They conclude that timely diagnosis and prompt surgical intervention provides excellent prognosis for both mother and foetus. According to Xu et al., once SHiP is diagnosed, exploratory laparotomy is to be performed. ${ }^{2}$ Whether LSCS is performed at the same time should be based on the conditions of operation and foetus. However, Brossens et al. suggests that in preterm cases where the bleeding points are successfully handled, there is a place for conservative management till term. ${ }^{5}$

Recently, Markou and Fysekidis suggested that in the absence of hypovolaemic shock or fetal distress, expectant management could be considered. ${ }^{6}$ The possibility that recovery can be complicated by the development of infected haematomas or recurrence of SHiP needs to be taken into account. However, successful surgical intervention does not rule out the recurrence of SHiP.?

The current patient remained stable despite the para-uterine haematoma and haemoperitoneum diagnosed by USS and MRI. She received blood transfusion, antibiotics and analgesics. Foetal status also remained stable. A CT angiography of the abdomen, taken after weighing the risks and benefits of imaging studies and obtaining consent, revealed no ongoing haemorrhage. ${ }^{8}$ Hence, she was conservatively managed.

During LSCS, the current patient was found to have a bicornuate uterus with placenta percreta at the junction of the two horns. The uterine anomaly and placenta percreta were not diagnosed in the antenatal period as the uterine indentation was shallow, the placenta percreta was focal, the uterine cavity was distended with the pregnancy and the right-sided haematoma became the main focus of attention on acute presentation.

The incidence of bicornuate uterus in a fertile population ranges from $0.1-0.6 \%$. It is believed to account for $20 \%$ of uterine anomalies. ${ }^{9}$ Bicornuate uterus (American Fertility Society Class IV) forms when the Mullerian ducts fuse incompletely at the level of the uterine fundus. The depth of the groove and the length of the uterine septum depends on the degree of fusion. ${ }^{10}$ Bicornuate uterus is associated with placenta accreta spectrum (PAS) disorders. The failure of normal decidualisation in the uterine area corresponding to the defect of the endometriummyometrial interface leads to the development of 
trophoblastic tissue deep within the myometrium and thus results in PAS disorders. ${ }^{11}$

In the current patient, the origin of haemoperitoneum may have been from bleeding from subserosal vessels of the uterus at the junction of the horns where the placenta was adherent. Later it may have resulted in the organised haematoma in the right para-uterine region.

\section{Conclusion}

Pregnant patients presenting with acute abdominal pain should be evaluated thoroughly before contemplating surgical procedure or delivery. To an extent, this can avoid surgical morbidity and iatrogenic prematurity. Spontaneous intraperitoneal haemorrhage in pregnancy is a rare but lifethreatening disease. In cases of endometriosis, assisted reproductive techniques, uterine anomalies and fibroids complicating pregnancy, SHiP is to be ruled out in patients presenting with acute abdominal pain. Foetal outcome depends on gestational age, hypovolaemia of the mother, the rapidity of diagnosis and aggressive management.

\section{AUTHORS' CONTRIBUTION}

JS and SA clinically managed the case. AK provided the radiology data. JS drafted the manuscript. LK, AK and SA critically reviewed the manuscript. All authors approved the final version of the manuscript.

\section{References}

1. Lier MCI, Malik RF, Ket JCF, Lambalk CB, Brosens IA, Mijatovic V. Spontaneous hemoperitoneum in pregnancy (SHiP) and endometriosis - A systematic review of the recent literature. Eur J Obstet Gynecol Reprod Biol 2017; 219:57-65. https://doi. org/10.1016/j.ejogrb.2017.10.012.

2. Xu Y, Zhou Y, Xie J, Yin X, Zhang X. Intraperitoneal hemorrhage during pregnancy and parturition: Case reports and literature review. Medicine (Baltimore) 2019; 98:e16300. https://doi. org/10.1097/MD.0000000000016300.

3. Van den Bosch T. Spontaneous haemoperitoneum in pregnancy (SHiP): Take-home messages. BJOG 2017; 124:313. https://doi. org/10.1111/1471-0528.14443.

4. Lier M, Malik RF, van Waesberghe J, Maas JW, van Rumpt-van de Geest DA, Coppus SF, et al. Spontaneous haemoperitoneum in pregnancy and endometriosis: A case series. BJOG 2017; 124:306-12. https://doi.org/10.1111/1471-0528.14371.

5. Brosens IA, Fusi L, Brosens JJ. Endometriosis is a risk factor for spontaneous hemoperitoneum during pregnancy. Fertil Steril 2009; 92:1243-5. https://doi.org/10.1016/j.fertnstert.2009.03.091.

6. Markou GA, Fysekidis M. Spontaneous hemoperitoneum during pregnancy. Is there any place for a wait and see approach in the absence of maternal hypovolemic shock or fetal distress? Eur J Obstet Gynecol Reprod Biol 2018; 225:260-1. https://doi. org/10.1016/j.ejogrb.2018.04.004.

7. Maya ET, Srofenyoh EK, Buntugu KA, Lamptey M. Idiopathic spontaneous haemoperitoneum in the third trimester of pregnancy. Ghana Med J 2012; 46:258-60.

8. Committee Opinion No. 723: Guidelines for Diagnostic Imaging During Pregnancy and Lactation. Obstet Gynecol 2017; 130:e210-16. https://doi.org/10.1097/AOG.0000000000002355.

9. Attaran M, Gidwani GP. Chapter 9 - Congenital and Developmental Anomalies. 200715 May 2009. In: Sokol AI, Sokol ER. General Gynecology. Maryland Heights: Mosby, 2007. pp. 205-23.

10. Amesse LS, Pfaff-Amesse T. Mullerian Duct Anomalies. From: https://emedicine.medscape.com/article/273534-overview Accessed: Feb 21.

11. Jauniaux E, Ayres-de-Campos D, Langhoff-Roos J, Fox KA, Collins S; FIGO Placenta Accreta Diagnosis and Management Expert Consensus Panel. FIGO classification for the clinical diagnosis of placenta accreta spectrum disorders. Int J Gynaecol Obstet 2019; 146:20-4. https://doi.org/10.1002/ijgo.12761. 